\title{
Optical networking special issue based on selected papers of IEEE ANTS 2015
}

\author{
Bheemarjuna Reddy Tamma ${ }^{1} \cdot$ Nabanita Das $^{2} \cdot$ Sudip Misra $^{3}$
}

Published online: 2 December 2016

(C) Springer Science+Business Media New York 2016

The ninth International Conference on Advanced Networks and Telecommunications-IEEE ANTS 2015 (http:// ants2015.ieee-comsoc-ants.org/), was held at Indian Statistical Institute (ISI) Kolkata, India, during December 15-18, 2015.

ANTS is a premier IEEE forum in the area of networking and telecommunications, held annually in India, financially sponsored by IEEE Communications Society. Over the last decade, ANTS has emerged as an internationally renowned platform for intense interaction between academia and industries around the world. A clear indicator of this fact is the large number of high-quality technical papers in the technical program, both from academia and industries, and their joint collaborations.

The technical program of ANTS 2015 consisted of 12 technical sessions in two parallel tracks with 61 contributory papers, six keynote talks, seven invited talks, four tutorials, three panels, a poster session, a demo/exhibit session and a Ph.D. forum. The ANTS 2015 Program Committee of 121 distinguished members worked hard to organize the technical program. Following a rigorous peer-review process, out of 178 submissions, only 52 long papers and nine short papers were accepted for presentation and publication in the

Bheemarjuna Reddy Tamma

tbr@iith.ac.in

Nabanita Das

ndas@isical.ac.in

Sudip Misra

Sudip.Misra@sit.iitkgp.ernet.in

1 IIT, Hyderabad, India

2 ISI, Kolkata, India

3 IIT, Kharagpur, India proceedings. Another eight papers were accepted for presentation in the poster session.

Based on the comments received from the reviewers, the authors of nine accepted papers in the area of optical networking were invited to submit an extended version of their work for possible publication in this Optical Networking Special Issue of the Springer Photonic Network Communications (PNET) journal. After a thorough review process, eight papers have been selected for publication. A brief summary on the accepted papers is given below.

In Priority-based content processing with Q-routing in Information Centric Networking (ICN), Sibendu Paul, Bitan Banerjee, Amitava Mukherjee and Mrinal K. Naskar address content management issue in a cache with finite storage capability in ICN by proposing an efficient content management policy that changes a router to a self-sustained cache. A novel algorithm based on Q-routing is proposed to determine the order of service for content packets in the buffer of a cache and find next node toward the destination with minimum propagation delay.

In SRAM-Based Novel Hardware Architecture for Longest Prefix Matching for IP Route Lookup, Sanchita Saha Ray, Surajeet Ghosh, and Bhaskar Sardar propose a novel, lowcost static random access memory (SRAM)-based hardware architecture for longest IP prefix matching which stores prefixes in the forwarding table and next hop information in a two-tier hierarchical SRAM-based memory structure: next hop global memory (NHGM) and next hop link memory (NHLM). It is reported that the average search throughputs achievable from this architecture are roughly 850 MLPS in IPv4 and 780 MLPS in IPv6.

In Clustering-based multi-hop protection scheme for longreach passive optical network against single shared-risk link group failure, Jitendra Gupta and Aneek Adhya propose a novel heuristic scheme to protect networks against single 
shared-risk link group (SRLG) failure in long-reach passive optical network (LR-PON) which ensures a certain degree of reliability (as determined by the network operator) to the users. In the proposed scheme, clustering-based multi-hop protection (CMHP), residual capacity of backup optical network units (ONUs) is allocated among the ONUs that require protection by using bypass-based multi-hop traffic transmission strategy through backup fibers. From simulation studies, it is reported that CMHP scheme reduces the total length of backup fiber significantly with reference to an existing scheme to protect against single SRLG failure in LR-PONs.

In Benefits of Sliceable Photonic-based Transponders in Metro and Long-Haul Networks, Sourav Das, Onur Turkcu, Anuj Malik, Pravin Mahajan and Biao Lu analyze benefits of photonic integration-based sliceable transponders (sliceable photonics) in terms of savings in line modules, efficient bandwidth utilization and power requirements compared to discrete optics transponder-based solutions (CFP/CFP2-C form-factor pluggable) in metro and long-haul networks. It shows that the sliceable photonics architecture reduces the number of line modules by half compared to discrete photonic-based transponder architecture in metro networks. In long-haul networks, the number of discrete optics-based $100 \mathrm{G}$ transponders required is five times that of sliceable photonics.

In MCRB for Timing, Phase and Frequency Estimation in Presence of Self-Phase Modulation for Low Rate Optical Communication, Debarati Sen derives the theoretical lower bound, namely Modified Cramer-Rao Bound (MCRB) for symbol timing, phase and frequency offset in the presence of nonlinear self-phase modulation (SPM) in a dispersion compensated long-haul coherent fiber link. It is found that SPM induces underdamped oscillation on the MCRB bounds depending on the pulse shapes (symmetric and asymmetric) utilized. Moreover, the MCRB for timing estimation is reduced due to the presence of SPM, but only when a prefilter with sufficiently large bandwidth is present.

In Designing a Green Optical Network Unit using ARMAbased Traffic Prediction for Quality of Service Aware Traffic, Chayan Bhar, Nilesh Chatur, Atri Mukhopadhyay, Goutam Das and Debasish Datta propose a novel MAC protocol for optical access networks (OANs) which ensures energy efficiency in the presence of QoS aware traffic. The proposed scheme incorporates auto-regressive and moving average (ARMA) model for predicting self-similar nature of Ethernet traffic in ONUs and selecting different sleep modes of operation of ONUs with significant energy efficiency and QoS figures.
In Cost and Energy Efficient Backhaul Options for Heterogeneous Mobile Network Deployments, Fabricio Farias, Matteo Fiorani, Sibel Tombaz, Mozhgan Mahloo, Lena Wosinska, Joao C. W. A. Costa and Paolo Monti propose a comprehensive methodology that can be used to analyze the total cost of ownership (TCO) of a number of backhaul options based on fiber, microwave and copper technologies for heterogeneous mobile network deployments in both greenfield and brownfield scenarios for the time period between years 2015 and 2025. From the study, it is reported that even though microwave and fiber will be predominately used in the future, the possible migration paths leading to such fiber- and microwave-based backhaul scenarios might be different, depending upon factors such as spectrum and license costs, time to deployment, availability of equipment and QoS requirements.

In Design and Analysis of a Resilient Packet Ring based Backhaul for LTE Networks that Reduces Handover Latency, Atri Mukhopadhyay and Goutam Das introduce a modified resilient packet ring (MRPR) based backhauling architecture to reduce handover latency when $\mathrm{X} 2$ interface is implemented over the optical backhaul for LTE networks. MRPR successfully implements X2 interface between LTE base stations over the fiber backhaul and then reduces the delay suffered by the packets that are transmitted over the X2 interface. Finally, it is recommended that the network load should be kept under control (less than 90\%) when MRPR is used as the LTE backhaul.

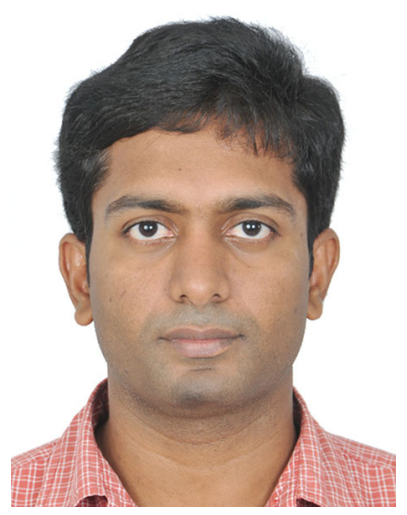

Bheemarjuna Reddy Tamma
is an Associate Professor and
the Head of the Department of
Computer Science and Engineer-
ing at IIT Hyderabad, India. He
obtained his M.E. from NIT
Rourkela in 2002 and Ph.D. from
IIT Madras, India, in 2007 and
then worked as a postdoctoral
fellow at the University of Cal-
ifornia San Diego (UCSD) divi-
sion of California Institute for
Telecommunications and Infor-
mation Technology (CALIT2)
prior to taking up faculty position at IIT Hyderabad, India, in 2010. His research interests are in the areas of converged cloud radio access networks, 5G, SDN, IoT/M2M, and Green ICT. He has published over 70 papers in refereed international journals and conferences. He is a member of IEEE and served as a TPC co-chair for IEEE ANTS 2015, a TPC vice chair for IEEE ANTS 2014, and a Ph.D. student forum co-chair for IEEE ANTS 2013 conferences. 


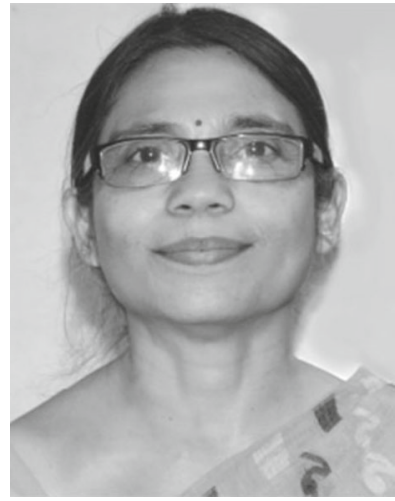

Nabanita Das received the B.Sc. degree in Physics in 1976, B.Tech. degree in Radio Physics and Electronics in 1979 from Calcutta University, M.Tech. in Computer Science in 1982, and Ph.D. in 1992 from Jadavpur University. She is currently a professor and Head of Advanced Computing and Microelectronics Unit of Indian Statistical Institute, Kolkata. She held visiting researcher positions in Paderborn University, Germany and San JoseState University. Her research interests include mobile computing, ad hoc and sensor networks, parallel and multicore computing. She has published more than 70 technical articles. She acted as the co-editor of Distributed Computing-IWDC 2004 LNCS, Springer, guest editor of special issue on Resource Management in Mobile Communication Networks of Microprocessors and Microsystems, 2004, Elsevier, editor of Recent Trends in High Performance Computing, Proc. of International Workshop on High Performance Computing, 1998-1999. She served as the Chair of Women in Engineering (WIE) affinity group of IEEE Calcutta section during 2009-2011. She is a senior member of the IEEE.

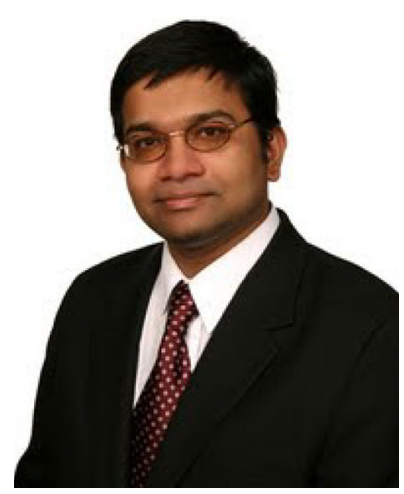

Sudip Misra is an Associate Professor in the Department of Computer Science and Engineering at the Indian Institute of Technology Kharagpur. Prior to this he was associated with Cornell University (USA), Yale University (USA), Nortel Networks (Canada) and the Government of Ontario (Canada). He received his Ph.D. degree in Computer Science from Carleton University, in Ottawa, Canada. He has several years of experience working in the academia, government, and the private sectors in research, teaching, consulting, project management, architecture, software design and product engineering roles.
His current research interests includes Wireless Ad Hoc and Sensor Networks, Internet of Things (IoT), Computer Networks, Learning Systems, and algorithm design for emerging communication networks. Dr. Misra is the author of over 260 scholarly research papers, including $140+$ reputed journal papers. He has won seven research paper awards in different conferences. Recently, he and his students won Samsung Innovation Award and the IEEE ComSoc Student Competition. He was also awarded the IEEE ComSoc Asia Pacific Outstanding Young Researcher Award at IEEE GLOBECOM 2012, Anaheim, California, USA. He was also the recipient of several academic awards and fellowships such as the Young Scientist Award (National Academy of Sciences, India), Young Systems Scientist Award (Systems Society of India), Young Engineers Award (Institution of Engineers, India), (Canadian) Governor General's Academic Gold Medal at Carleton University, the University Outstanding Graduate Student Award in the Doctoral level at Carleton University and the National Academy of Sciences, India - Swarna Jayanti Puraskar (Golden Jubilee Award).

Dr. Misra was also awarded the Canadian Government's prestigious NSERC Post Doctoral Fellowship and the Humboldt Research Fellowship in Germany. Dr. Misra has been serving the editorial boards of distinguished journals such as the International Journal of Communication Systems (Wiley) and the IET Wireless Sensor Systems (UK). In the past, he served as the Associate Editor/Editorial Board Member of the Telecommunication Systems Journal (Springer), Security and Communication Networks Journal (Wiley), and the EURASIP Journal of Wireless Communications and Networking, IET Communications Journal, and the Computers and Electrical Engineering Journal (Elsevier).

Dr. Misra has published 9 books in the areas of wireless ad hoc networks, wireless sensor networks, wireless mesh networks, communication networks and distributed systems, network reliability and fault tolerance, and information and coding theory, published by reputed publishers such as Cambridge University Press, Springer, Wiley, and World Scientific.

Dr. Misra was invited to chair several international conference/workshop programs and sessions. He served in the program committees of several international conferences. He was also invited to deliver keynote/invited lectures in over 30 international conferences in USA, Canada, Europe, Asia and Africa. 